Theoretical Aspects of Electroweak and Other Interactions in Medium Energy Physics - Interim Progress Report

(Nov. 20, 1992)

DOE/ER/ 40448--5

Nimai C. Mukhopadhyay

DE93 003714

Department of Physics, Rensselaer Polytechnic Institute

Troy, New York 12180-3590

\begin{abstract}
In the project period under review, we have maintained progress in the study of electroweak structure of baryon resonances, and in the analysis of data for pion and eta photoproduction. Four graduate students are currently associated with the program. One has obtained his Ph.D. degree in the year under review. Six research articles have been completed in this year and five conference contributions have been made. Collaborations with scientists from Illinois, Los Alamos, Westinghouse, William and Mary, Yale, Mainz (Germany), Saskachewan (Canada) and TRIUMF (Canada) continue, along with participation in colleborations at CEBAF.
\end{abstract}

\title{
DISCLAIMER
}

This report was prepared as an account of work sponscred by an agency of the United States Government. Neither the United States Government nor any agency thereof, nor any of their employees, makes any warranty, express or implied, or assumes any legal liability or responsibility for the accuracy, completeness, or usefulness of any information, apparatus, product, or process disclosed, or represents that its use would not infringe privately owned rights. Reference herein to any specific commercial product, process, or service by trade name, trademark, manufacturer, or otherwise does not necessarily constitute or imply its endorsement, recommendation, or favoring by the United States Government or any agency thereof. The views and opinions of authors expressed herein do not necessarily state or reflect those of the United States Government or any agency thereof. 
1. Introduction

The research done under this U.S. Dept. of Energy Grant in the current project year has hacl its primary focus on the theory of electroweak excitation of baryon resonances, in particular, in the context of the continuous electron beam accelerators, currently under development at CEBAF, and in operation around the world at research centers like Mainz in Germany, and Amsterdam, in the Netherlands. These research interests have also been the prime motivations of the travels of the principal investigator (NCM) to these centers of research in this grant year.

Below we first give in Section 2 a summary of the progress of our research in the grant year April 1992 to March 1993. Graduate students' progress in this period is discussed in Section 3. Section 4 deals with the research travel of the principal investigator. Section 5 summarizes current external collaborations. Section 6 gives other research news in the context of this grant. Appendix A lists students working with this program. Appendix B details the invited presentations of the principal investigator in the current grant year. Appendix C lists the papers completed in the academic year 1992-93.

References cited herein are collected in the Section 7, following Section 6.

\section{Research Progress}

Six paper [1-6] have been completed or published during this project year. Five conference contributions [7-11] have been also made during this period. One Ph.D. thesis [12] has been written. Below is a brief review of these research items. 
(1) Relations between pion photoproduction and Comptor: scattering in the Delta (1232) region

M. Benmerrouche and NCM $[1,3,7]$ have examined the two-channel problem with the diagonal channels $\pi N \rightarrow \pi N, \gamma N \rightarrow \gamma N$, and their relations to the off-diagonal process $\gamma N \rightarrow \pi N$. Using the experimental analysis of Grushin et al. [13] giving independently real and imaginary parts of pion photoproduction amplitudes, we show that the unitarity yields a precise value for the Compton amplitude

$$
\operatorname{Im} f_{M M}^{1+}=(15.4 \pm 0.21) \times 10^{-4} / m_{\pi^{+}}
$$

for the photon lab energy of $E_{\gamma}=348 \mathrm{MeV}$. From the data on total photohadron crosssection, we can extract, using optical theorem, the imaginary part of the forward Compton amplitude, and compare with what we get from the analysis above. Their agreement gives a measure of confidence to the pion photoproduction data set.

We also analyze the prospect of the determination of the resonant $M 1$ and $E 2$ amplitudes [14] in the $N \leftrightarrow \Delta$ transition using polarized photons [15] at Brookhaven, Mainz and other photon facilities.

(2) Paramagnetic polarizability of the proton

From low-energy Compton scattering experiments it has been possible to infer values of electric and magnetic polarizabilities of the proton [16]. Of these, the latter is hard to measure and difficult to interpret. A.M. Nathan (University of Illinois), L. Zhang and $\operatorname{NCM}[2]$ show that the contribution of the virtual excitation of the Delta resonance $\beta_{p}^{\Delta}$ 
'can be reliably computed from the pion photoproduction dita:

$$
\beta_{p}^{\Delta}=(13 \pm 3) \times 10^{-4} \mathrm{fm}^{3}
$$

while the paramagnetic polarizability inferred from the low-energy Compton scattering experiment is $[16]$

$$
\beta_{p}=(15 \pm 3) \times 10^{-4} \mathrm{fm}^{3} \text {. }
$$

Thus, the Delta resonance saturates the quantity in (3). This has interesting implications in the interpretation of this quantity in the chiral perturbation theory [6].

(3) The Bloom-Gilman duality and the disappearing Delta resonance

The relationship between the resonance excitation and deep inelastic scattering with electroweak probes for hadrons is called duality [17]. Bloom and Gilman [18] (BG) have discovered two specific empirical relations in the context of inelastic electron scattering, which are called together the BG Duality. Stoler [19] has pointed out that one of these two relations, the resonance falling roughly at the same rate as the background underneath it, as a function of the momentum transfer squared $Q^{2}$, fails for the Delta resonance. This has prompted C.E. Carlson (College of William and Mary) and NCM $[4,10]$ to examine the dual relationship between resonances and scaling curves for inelastic structure function $\nu W_{2}$.

We use the Stoler database for $Q^{2}$ in the range from 1 to $17(\mathrm{GeV} / \mathrm{c})^{2}$. We find that the structure function $\nu W_{2}$, averag'd o 'er suitable ranges of the Bjorken variable $x$, yields the 
'smooth scaling curve seen at higher values of $Q^{2}$ at the same value of $x$, for all resonance regions. The Delta (1232) is no exception to this rule, despite the strange disappearance of it at higher $Q^{2}$, emphasized by Stoler.

(4) Dynamic symmetry in meson physics:

F. Iachello (Yale University), L. Zhang and NCM have examined earlier [20] the role of the dynamic symmetry $(D S) U(4) \supset S O(4)$ as a representative of the property of the $q \bar{q}$ color string. The success of this approach has encouraged us to proceed with a careful examination of the possible problems that we may encounter. The DS here is only approximate, as in nuclear and molecular physics.

In this work [5], we have discovered that there can be only three spectroscopic classes or intervals for the color vibrational degree of freedom. Depending on the group characteristics of these intervals, we call these the $O, U$ and $N$ types, these being $S O(4), U(3)$ or equidistant (neutral) types.

We are currently investigating the frequencies of various spectroscopic intervals and other underlying dynamical consequences. A puzzle that we have discovered [5] is the non-occurence of the $N$-type intervals, even as we expect them to be most frequent for the $U(4) \supset S O(4)$ DS, with the group representation quantum number $N$ being very large $(N \rightarrow \infty)$, representing the quark confinement.

(5) Recent progress in baryon spectroscopy

The Particle Data Group (PDG) have brought out a new edition of the particle data compilation in June 1992 [21]. The principal investigator has been invited at the CEBAF Workshop in the summer of 1992 to report on this, and has prepared a summary of 
'the highlights of PDG-92 pertaining to the physics interests of CABAF [6], particularly focussing on the nucleon, Delta (1232), Roper (1440) and $S_{11}$ (1535). A crucial issue of considerable topical interest is the nature of the Roper [22]. Carlson and NCM [23] have discovered clear-cut tests in the longitudinal and transverse structure functions, providing a way of testing the hypothesis of the Roper (1440) resonance having a hybrid structure (i.e., not a three-quark structure as assumed in ordinary quark models).

(6) Electromagnetic excitation of the $S_{11}(1535)$ resonance in the eta photo- and electroproduction

The process $\gamma+p \rightarrow p+\eta$, where $\gamma$ is either a real or a virtual photon, is being studied experimentally at Bates, Mainz and Bonn, and is a prime candidate for study at CEBAF with the CLAS spectrometer. From the available real photon data, Benmerrouche and the author (BM) have extracted the product [24]

$$
\Gamma_{\eta}^{1 / 2} A_{1 / 2}=(26 \pm 3) \times 10^{-3}
$$

where $\Gamma_{\eta}$ is the $\eta N$ width of the $S_{11}(1535)$ and $A_{1 / 2}$ is the $p \rightarrow S_{11}$ photon helicity amplitude. In a contribution to the Baryons-92 Conference, $\mathrm{BM}[8]$ have extracted a quantity

$$
A=\sqrt{x \Gamma_{\eta}} A_{1 / 2} / \Gamma_{T}
$$

where $x$ is a kinematic factor, $\Gamma_{T}$ is the total width of the $S_{11}$ (1535). The quantity $A$ is determined as a function of $Q^{2}$. This is of interest from the point of view of hadron models. 
'Benmerrouche has examined the model dependence of this extraction, on the basis of the existing world data base, in his thesis [12]. Further work on this is continuing.

(7) The Skyrme model approach to the baryon structure

The recent work by the graduate student Lie-Sheng Zhang and NCM has focused on the Skyrme approach to the electroweak structure on the nucleon and the Delta resonance. A progress report has been given at the Yale Baryon-92 conference [9]. At issue here is the idea that the constraints of meson (and glueball) physics are enough to describe properties of baryons, which emerge as solitons in this theory. We are attempting to go beyond the latest progress on this approach by the Syracuse and Bern groups [25] and constructing a theory that respects the conservation of the vector and axial- vector current, to the leading order in the inverse of number of colors $\left(N_{c}^{-1}\right)$, an expansion advocated by Witten. Our efforts are continuing in the context of a realistic description of the known properties of the nucleon and the Delta [9].

(8) Physics beyond the standard model

P. Herczeg (Los Alamos) and NCM have been exploring the nuclear muon capture process with a view to develop a strategy to search for possible violation of time reversal invariance. In the standard model, such violation should be unobservable, while several extensions of the standard model would allow for observable $T$ violation. Specific case studies in ${ }^{12} \mathrm{C}$ and ${ }^{16} \mathrm{O}$ are made in a recent conference progress report [11].

3. Progress of students associated with the program

In the current program year, five graduate students and one undergraduate have been associated with this program. One of them, M. Benmerrouche has been a full time re- 
'search' assistant with the program. He has completed his Ph.D. dissertation entitled Photo- and Electroproduction of Eta Mesons in the $S_{11}$ (1535) Region in the summer of 1992. For this work, he has been rewarded with the Huntington Prize of the RPI Physics department. He, and the other full-time research assistant, Lie-Sheng Zhang, have also been awarded financial assistance to attend the Baryon-92 Conference at Yale University by the Organizing Committee of the Yale conference. They have coauthored with NCM three papers contributed to the Baryon-92 Conference [7-9]. Benmerrouche has coauthored two papers on Compton scattering [1,3], while the papers on nucleon polarizability $[2]$ and dynamic symmetry [5] have been coauthored by Zhang. After graduation, Benmerrouche has left to join a postdoctoral appointment at the Saskachewan Accelerator Lab. The third graduate student in this program, K-C. Yeong, has joined our program in the summer of 1992, after passing his Ph.D. Qualifying Examination, as a full-time research assistant. Graduate students Richard Schelp and Ernesto O. Ulloa, and Erik Saperstein, an undergraduate, are also currently associated with the program as unpaid assistants. They are currently supported by the RPI Physics Department. In due course, they will be hopefully supported by the continuation of this grant. The support for the undergraduate physics major comes from a special RPI program.

Further information on students can be found in Appendix A.

4. Research travel of the principal investigator

A list of outside institutions where the principal investigator has travelled in the present year to give seminars or colloquia, on the research covered by the Grant, is appended (Appendix B). 
Important domestic research travels of NCM have been to the Institute of Nuclear Theory (INT), Seattle, between May 3 and May 23 of this year, to attend the program on Fundamental Symmetries in Nuclei (Program 7 of the INT); Brookhaven National Laboratory, between May 28 and May 29, to attend the Workshop on Hadron Structure from Photo-reactions at Intermediate Energies (accompanied by the graduate student Benmerrouche); Yale University, between May 31 and June 4, to attend the international conference, Baryons- 92, (accompanied by the graudate students Benmerrouche, Yeong and Zhang); CEBAF, between June 13 and June 20, to attend the summer workshop.

The main foreign trip by NCM has been to the Johannes Gutenberg University, Mainz, Germany, as a Visiting Professor during the summer semester, '92. This sojourn has also included short research trips to Bonn (Germany), NIKHEF-K in Amsterdam (Holland), Catholic University in Louvain (Belgium), Wroclaw University in Wroclaw (Poland).

\section{Current external collaborations}

Current collaborations of this research program with outside scientists include those with former graduates of this program. These are extremely valuable and provide continuity of research. Alumni of this program maintaining active research collaboration are: Dr. M. Benmerrouche (Saskachewan Acc. Lab., Canada) $\left[S_{11}\right.$ (1535) electroexcitation]; Dr. R. Davidson (Johannes Gutenberg Univ., Mainz, Germany) and Dr. R. Wittman (Westinghouse Lab, South Carolina) [Delta (1232) Physics]; Dr. B. Doyle (TRIUMF, Canada) [Nuclear weak interaction]. NCM is also collaborating with Prof. F. Iachello (Yale) on dynamic symmetry; Prof. C. Carlson (College of William and Mary) on perturbative QCD; Dr. P. Herczeg (LANL) on nuclear weak interaction; Prof. A. Nathan (Illinois) on 
'Compton scattering.

6. Other research news related to the current program

Progress of this research has been featured in the October issue of the Rensselaer Magazine "Review" issue with a picture of the principal investigator and a story on his research. NCM has been on the International Advisory Committee of the international conference, Baryons-92, and on the Organizing Committee of the CEBAF Summer Workshop of 1992. His association with the Excited Baryons Collaboration at CEBAF is continuing.

\section{References cited}

[1] M. Benmerrouche and N.C. Mukhopadhyay, Phys. Rev. D46, 101(1992).

[2] N.C. Mukhopadhyay, A.M. Nathan and L. Zhang, Phys. Rev. $\underline{\text {, }}$ Rapid Communications (in press).

[3 ] N.C. Mukhopadhyay and M. Benmerrouche, "Connections between Compton Scattering and Pion Photoproduction in the Delta Region", Invited paper, in the Proceedings of the Workshop on Hadron Structure from Photoreactions at Intermediate Energy (A.M. Nathan and A.M. Sandorfi, editors), Report \# BNL47972, p. 117 (1992).

[4 C.E. Carlson and N.C. Mukhopadhyay, "Duality in the Inelastic Structure Function and the Disappearing Delta Resonance”, WM-92-108/RPI-N79-92, submitted to Phys. Rev. Lett.

[5 ] N.C. Mukhopadhyay and L. Zhang, "Dynamic Symmetry in Hadron Physics: A 
Mesonic Puzzle for the $U(4) \supset S O(4)$ Chain", Invited paper, Symmetry and Physics (B. Gruber, editor), Plenum Press (in press).

[6 ] N.C. Mukhopadhyay, "Recent Progress in Baryon Spectroscopy", Mainz Report \# MKPH-T-92-14, Invited Paper, to appear in the Proc. of the CEBAF Summer 1992 Workshop (F. Gross et al., editors), Am. Inst. Physics.

[7 ] M. Benmerrouche and N.C. Mukhopadhyay, "Relationships between Pion Photoproduction and Compton Scattering in the $\Delta$ (1232) Region", contribution to the International Conf. on the Structure of Baryons and Related Mesons (hereafter, BARYONS-92), Yale Univ., Volume on contributed papers (M. Gai et al., editors), p. 26.

[8 ] M. Benmerrouche and N.C. Mukhopadhyay, "Electromagnetic Transition Form Factor of $\gamma p \rightarrow S_{11}(1535)$ from $\eta$ Electroproduction", BARYONS-92, p. 12.

[9] N.C. Mukhopadhyay and L. Zhang, "Electroweak Nucleon to Delta Transition Properties in the Realistic Skyrme Model", BARYONS-92, p. 72.

[10 ] C.E. Carlson and N.C. Mukhopadhyay, "Bloom Gilman Duality and Disappearing Delta Resonance”, BARYONS-92, p. 143.

[11 ] P. Herczeg and N.C. Mukhopadhyay, "Theory of T-violation in Muon Capture Processes", contribution to the Int'l conf. on Weak and Electromagnetic Interactions in Nuclei (WEIN-92), Dubna, Russia, 1992. 
[12 $]$ M. Benmerrouche, "Photo- and Electroproduction of $\eta$ Mesons in the $S_{11}$ (1535) Region", Ph.D. Thesis, Rensselaer Polytechnic Institute, Troy, NY (June 1992), unpublished. (See abstract in Appendix D).

[13 ] V.F. Grushin et al., Sov. J. Nucl. Phys. $\underline{38}, 881$ (1983).

[14 ] R.M. Davidson, N.C. Mukhopadhyay and R.S. Wittman, Phys. Rev. D43, 71 (1991).

[15 ] G. Blanpied et al., Phys. Rev. Lett. $\underline{69}, 1880$ (1992).

[16 ] F.J. Federspiel et al., Phys. Rev. Lett. 67, 1511 (1991); A. Zieger et all., Phys. Lett. B278, 23(1992).

[17] R. Dolan, D. Horn and C. Schmid, Phys. Rev. $\underline{166}$, 1768 (1968).

[18 ] E.D. Bloom and F.J. Gilman, Phys. Rev. Lett. $\underline{25}, 1140$ (1970).

[19] P. Stoler, Phys. Rev. Lett. $\underline{66}, 1003$ (1991).

[20 ] F. Iachello, N.C. Mukhopadhyay and L. Zhang, Phys. Rev. D44, 398 (1991).

[21 ] K. Hikasa et al. (Particle Data Group), Phys. Rev. D45, I.1 (1992).

[22] Z. Li, V. Burkert and Z. Li, Phys. Rev. D46, 70 (1992).

[23 ] C.E. Carlson and N.C. Mukhopadhyay, Phys. Rev. Lett. $\underline{67}, 3745$ (1991).

[24] M. Benmerrouche and N.C. Mukhopadhyay, Phys. Rev. Lett. $\underline{67}, 1070$ (1991). 
[25 ] P. Jain et al., Phys. Rev. D37, 3252 (1988); U.-G. Meissner et al., ibid D39, 1956 (1989). 
Appendix A: Student collaboration with the program, 1992-93

- M. Benmerrouche ${ }^{1}$. Graduate student

* L. Zhang. Graduate student

* K.C. Yeong. ${ }^{2}$ Graduate student

†.O. Ulloa. Graduate student

E. Saperstein. Undergraduate student

- Research assistant supported by this grant.

t RPI support. Waiting for the DOE support

Independent support

1 With this program through Aug. '92

${ }^{2}$ DOE support began in Summer '92 
Appendix B: Principal Investigator's host institutions for invited seminars, research presentations, colloquia, etc. in 1992-93 dealing with reports on projects under the DOE grant

(1) Univ. of $\mathrm{K}$ ?ntuky, Lexington, $\mathrm{KY}$

(2) Univ. of Massachussets, Amherst, MA

(3) Institute for Nuclear Theory, Univ. of Washington, Seattle, WA

(4) Brookhaven National Lab, Upton, NY

(5) BARYON-92 Conf., Yale Univ., New Haven, CT

(6) CEBAF Summer Workshop, Newport News, VA

(7) Particle Physics Seminar, Univ. of Bonn, Germany

(8) Inst. of Nuclear Physics, J. Gutenberg Univ., Mainz, Germany

(9) Inst. of Nucl. Physics (NIKHEF-K), Amsterdam, Holland

(10) Inst. de Physique Corpusculaire, U. de Louvain, Louvain-la-Neuve, Belguim

(11) Inst. for Theor. Phys., Wroclaw Univ., Wroclaw, Poland

(12) Univ. of Pittsburgh, Pittsburgh, PA

(13) Ohio Univ., Athens, OH

(14) VPI, Blacksburg, VA 
- Appendix C: Papers, conference contributions by the principle investigator and his students, wihtout or with external collaborators, completed/published in 1992 , under the current program.

Six research articles [1-6] listed in the References cited section and five conference contributions [7-11]. One Ph.D. thesis [12] finished in 1992.

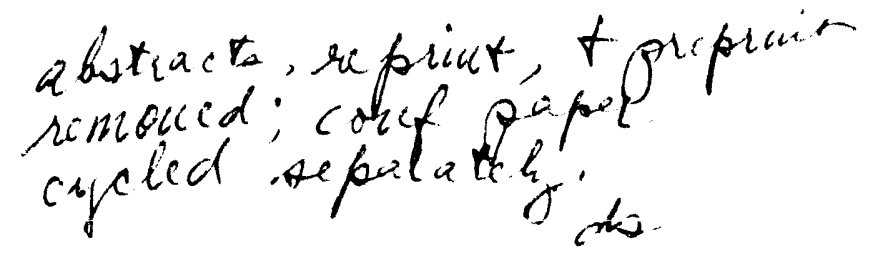



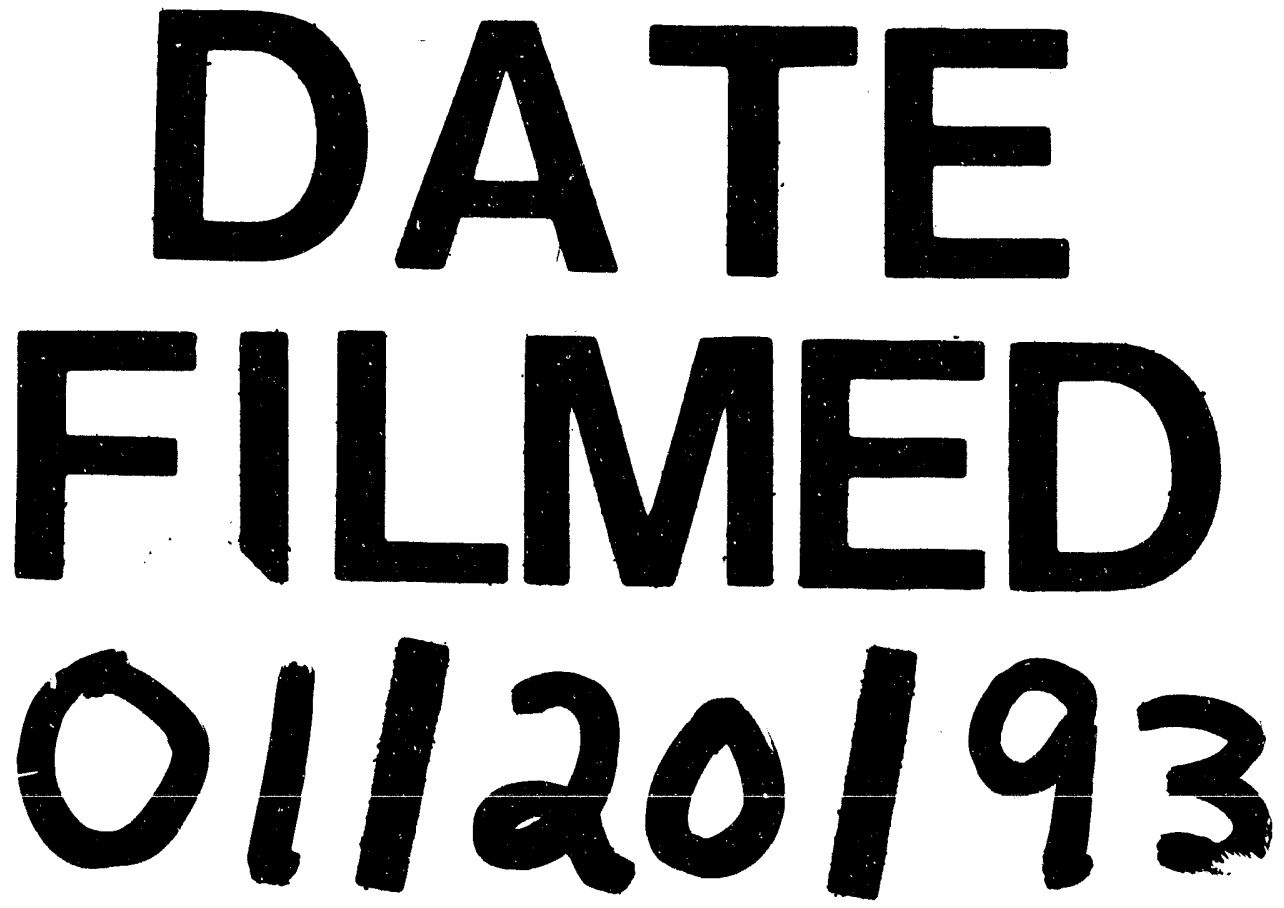
